\title{
THE FILTRATION EQUATION IN A CLASS OF FUNCTIONS DECREASING AT INFINITY
}

\author{
D. EIDUS AND S. KAMIN
}

(Communicated by Barbara L. Keyfitz)

\begin{abstract}
We deal with the Cauchy and external boundary problems for the nonlinear filtration equation with variable density. For each density we define a class $\phi$ of initial functions $\varphi$, such that for any $\varphi \in \phi$ the problem is uniquely solvable in some set of functions decreasing at infinity with respect to space variables.
\end{abstract}

It is well known that the unique solvability of the Cauchy problem for the heat equation

$$
\rho u_{t}=\Delta u
$$

with density $\rho=$ const takes place in classes of increasing functions. This means that if an initial function $\varphi$ belongs to some class $\phi$ of functions increasing at infinity, then there exits a unique solution $u$ of the corresponding Cauchy problem, belonging to $\phi$ for any $t>0$. An analogous result is valid for the nonlinear filtration equation in the case $\rho=$ const (see $[1,5]$ ). However, for $\rho=\rho(x)$ it is, generally speaking, incorrect. It was shown in [4] that if $\rho$ tends to zero fast enough as $|x| \rightarrow \infty$ and $\varphi \in L^{\infty}\left(\mathbb{R}^{n}\right), n \geq 3$, then the Cauchy problem can have more than one solution belonging to the class $L^{\infty}\left(\mathbb{R}^{n}\right)$ for $t>0$. Nevertheless, the unique solvability is valid here in a more restricted class of solutions, namely in a class of functions decreasing fast enough as $|x| \rightarrow \infty$. It is essential that the behaviour of solutions for $t>0$ as $|x| \rightarrow \infty$ is different from that of initial functions.

Here we extend the results of [4] to the case of arbitrary $\rho(x)>0$ and initial functions $\varphi$ not necessarily belonging to $L^{\infty}\left(\mathbb{R}^{n}\right)$. We also deal with an external boundary problem. In order to include the above-mentioned case, where $\rho \rightarrow 0$ fast enough at infinity, we are looking for a solution in a class of functions decreasing as $|x| \rightarrow \infty$ in some integral sense. It turns out that initial functions can belong to a more general class. Thus, due to the nature of the problem, unlike [1], our class of unique solvability is different from the class of initial functions. Let us give a precise formulation.

We are looking for a nonnegative solution $u(x, t)$ of the following problem on the domain $Q_{T}:=\Omega \times(0, T), T>0$, where $\Omega$ is the exterior of an

Received by the editors January 16, 1992 and, in revised form, June 29, 1992.

1991 Mathematics Subject Classification. Primary 35K55, 35K65. 
$(n-1)$-dimensional smooth closed surface $\partial \Omega$ in the space $\mathbb{R}^{n}, n \geq 3$ :

$$
\begin{aligned}
\rho(x) u_{t} & =\Delta G(u), & & (x, t) \in Q_{T}, \\
\left.u\right|_{t=0} & =\varphi(x), & & x \in \Omega, \\
\left.u\right|_{\partial \Omega} & =0, & & t \in(0, T) .
\end{aligned}
$$

In this problem $\varphi \geq 0$ on $\Omega, \rho, \varphi \in L^{\infty}(\Omega(R)), \rho(x) \geq C(R)>0$ on $\Omega(R)$ where $\Omega(R)=\{x: x \in \Omega,|x|<R\}$, for all large enough $R>0$,

$$
\int_{\Omega} \frac{\rho \varphi}{|x|^{n-2}} d x<\infty,
$$

$G(u)$ is a smooth function for $u \geq 0$,

$$
\begin{gathered}
G(0)=0, \\
G^{\prime}(u)>0 \quad \forall u>0, \\
G(u) \geq \gamma u \quad \forall u \geq 1,
\end{gathered}
$$

and $\gamma=$ const $>0$. Moreover, $G^{\prime}(u)$ is increasing in some $0<u<\delta$ if $G^{\prime}(0)=0$.

Our main result: under the imposed restrictions the problem (1), (2), (3) has a unique nonnegative solution, satisfying the following condition:

$$
\lim _{R \rightarrow \infty} R^{1-n} \int_{S(R)} U(x) d s=0,
$$

where $S(R)=\{x: x \in \Omega,|x|=R\}$,

$$
U(x)=\int_{0}^{T} G(u(x, t)) d t .
$$

This is valid also for the Cauchy problem (1), (2), where $\Omega=\mathbb{R}^{n}$. We assume below that the Cauchy problem is a special case of (1), (2), (3), where $\Omega=\mathbb{R}^{n}$, $\partial \Omega=\varnothing$.

Thus, if $\rho \rightarrow 0$ fast enough as $|x| \rightarrow \infty$, then $\varphi(x)$ can increase for large $|x|$, provided that (4) remains valid. Then the solution of (1), (2), (3) is decreasing as $|x| \rightarrow \infty$ in the integral sense of (8).

The most important special case of (1) is the equation

$$
\rho(x) u_{t}=\Delta\left(u^{m}\right), \quad m>1 .
$$

Separating variables in (9), we get the stationary equation

$$
-\Delta\left(w^{m}\right)=\frac{1}{(m-1)} \rho(x) w .
$$

The recent paper [2] is devoted to the existence and uniqueness of a nontrivial nonnegative solution of $(10)$ in $\mathbb{R}^{n}, n \geq 3$. Let us notice that the asymptotics as $t \rightarrow \infty$ of the solution of the Cauchy problem (9), (2) has the form $w(x) \zeta(t)$, where $w$ is the solution of (10). We hope to give later a proof and possible generalizations for this statement. 
Suppose $R \geq R_{0}>0$, where $R_{0}$ is chosen so that the ball $|x|<R_{0}$ contains the surface $\partial \Omega$. Set

$$
\begin{gathered}
\mathbb{R}_{+}=\{\xi: \xi \in \mathbb{R}, \xi \geq 0\}, \\
S_{T}(R)=\left\{(x, t): x \in \mathbb{R}^{n},|x|=R, 0<t<T\right\}, \\
\Omega\left(R_{1}, R_{2}\right)=\left\{x: x \in \Omega, R_{1}<|x|<R_{2}\right\}, \\
Q_{T}(R)=\Omega(R) \times(0, T), \quad T>0, \\
\tilde{L}_{\mathrm{loc}}^{m}(\Omega)=\left\{v: v \in L^{m}(\Omega(R)) \forall R>R_{0}\right\}, \quad 1 \leq m \leq \infty, \\
\widetilde{L}_{\mathrm{loc}}^{m}\left(Q_{T}\right)=\left\{u: u \in L^{m}\left(Q_{T}(R)\right) \forall R>R_{0}\right\}, \quad 1 \leq m \leq \infty .
\end{gathered}
$$

Let $\tilde{C}_{0}^{\infty}\left(Q_{T}(R)\right)$ be a set of functions $g(x, t)$ belonging to $C^{\infty}\left(Q_{T}(R)\right) \cap$ $C\left(\overline{Q_{T}(R)}\right)$, such that

$$
\left.g\right|_{\partial \Omega}=0,\left.\quad g\right|_{S(R)}=0,
$$

for any $t \in(0, T)$, and $g=0$ if $t>T-\varepsilon$, where $\varepsilon=\varepsilon(g) \in(0, T)$.

Analogously, $\widetilde{C}_{0}^{\infty}\left(Q_{T}\right)$ will be a set of functions $g(x, t)$ belonging to $C^{\infty}\left(Q_{T}\right) \cap C\left(\bar{Q}_{T}\right)$ satisfying the first of conditions (11) and such that $g=0$ if $t>T-\varepsilon(g)$ or $|x|>R(g)$, where $\varepsilon(g) \in(0, T), R(g)>R_{0}$.

Later on we shall use a weak solution $u_{R, \theta}$ of equation (1) on the domain $Q_{T}(R)$, which satisfies the initial condition (2) for $x \in \Omega(R)$ and boundary conditions (3) and

$$
\left.u\right|_{S_{T}(R)}=\theta
$$

where $\theta \in L^{\infty}\left(S_{T}(R)\right), \theta \geq 0$ a.e. on $S_{T}(R)$. Then $u_{R, \theta} \in C\left(Q_{T}(R)\right) \cap$ $L^{\infty}\left(Q_{T}(R)\right)$ and for any $g \in \widetilde{C}_{0}^{\infty}\left(Q_{T}(R)\right)$ we have

$$
\begin{aligned}
& \int_{Q_{T}(R)}\left(G\left(u_{R, \theta}\right) \Delta g+\rho g_{t} u_{R, \theta}\right) d x d t+\int_{\Omega(R)} \rho \varphi g d x \\
& =\int_{S_{T}(R)} G(\theta) g_{\nu} d S_{T}(R),
\end{aligned}
$$

where $\nu$ is an external normal on $S_{T}(R)$. The existence of such solutions can be proved by an approximating procedure (see, e.g., $[6,1,4])$ using the results of [3].

For $\theta=0$ we set $u_{R, 0}=u_{R}$.

Definition 1. A weak solution of the problem (1), (2), (3) is a nonnegative function $u \in \widetilde{L}_{\text {loc }}^{1}\left(Q_{T}\right)$ satisfying

$$
U \in \tilde{L}_{\mathrm{loc}}^{\infty}(\Omega),
$$

where $U$ is as in (8),

(B)

$$
\int_{Q_{T}}\left(G(u) \Delta g+\rho u g_{t}\right) d x d t+\int_{\Omega} \rho \varphi g(x, 0) d x=0 \quad \forall g \in \tilde{C}_{0}^{\infty}\left(Q_{T}\right),
$$

and

(C) there exist such sequences $R_{m}, \theta_{m}, R_{m}>R_{0}, \theta_{m} \in L^{\infty}\left(S_{T}\left(R_{m}\right)\right)$, $m=1,2, \ldots$, that $\theta_{m} \geq 0$ for $(x, t) \in S_{T}\left(R_{m}\right)$ and $u_{R_{m}, \theta_{m}} \rightarrow u$ a.e. on $Q_{T}$ as $m \rightarrow \infty$. 
Note. By the comparison principle we obtain, using condition $(\mathrm{C})$, that

$$
u_{R} \leq u \quad \forall R \geq R_{0},
$$

a.e. on $Q_{T}(R)$.

Remark 1. If $u \in L_{\text {loc }}^{\infty}\left(Q_{T}\right)$, then condition (C) is obvious. In particular, if $\varphi \in L^{\infty}(\boldsymbol{\Omega})$, then a function $u \in L^{\infty}\left(Q_{T}\right)$ is a weak solution if it satisfies condition (B) (cf. [4]).

Below we use the word "solution" instead of "weak solution".

Definition 2. A solution $u$ of the problem (1), (2), (3) is minimal if for any solution $v$ of this problem

$$
u \leq v
$$

a.e. on $Q_{T}$.

Later on, $\eta(z), \eta: \mathbb{R} \rightarrow \mathbb{R}$, will be a function chosen so that $\eta \in C^{\infty}(\mathbb{R})$, $0 \leq \eta \leq 1$ on $\mathbb{R}$, where $\eta=0 \forall z \geq 1, \eta=1 \forall z \leq 0$, and $\eta^{\prime} \leq 0 \forall z \in \mathbb{R}$. For $\varepsilon \in(0, T), \tau \in(0, T-\varepsilon), R>R_{0}$, set

$$
\begin{gathered}
\eta_{\varepsilon \tau}(t)=\eta\left(\frac{t-\tau}{T-\varepsilon-\tau}\right), \\
V_{R}(r)=\eta\left(\frac{r}{R}-1\right), \quad r=|x|, \quad x \in \mathbb{R}^{n} .
\end{gathered}
$$

The following lemma is known (see e.g., [2, Lemma A.4]).

Lemma. Suppose $n \geq 3, \mu: \mathbb{R}^{n} \rightarrow \mathbb{R}^{+}, \mu \in \widetilde{L}_{\text {loc }}^{\infty}\left(\mathbb{R}^{n}\right), \mu|x|^{2-n} \in L^{1}\left(\mathbb{R}^{n}\right)$, and $U=|x|^{2-n} * \mu$. Then the function $U$ satisfies condition (8).

Theorem 1. Suppose $n \geq 3$, the function $G: \mathbb{R}_{+} \rightarrow \mathbb{R}$ satisfies the conditions (5), (6), (7), $\rho, \varphi$ are as above, and (4) is valid. Then there exists a minimal solution of the problem (1), (2), (3) satisfying condition (8).

Proof. Let $y$ be a fixed point in $\Omega(R)$, where $R>R_{0}$, and let $h_{m}$ be the solution of the following problem on $\Omega(R)$ :

$$
\begin{aligned}
& \Delta h=-\delta_{m}(x, y), \\
& \left.h\right|_{\partial \Omega}=\left.h\right|_{S(R)}=0,
\end{aligned}
$$

where $m=1,2, \ldots, \delta_{m}(x, y)$ as a function of $x$ is chosen so that $\delta_{m} \geq 0$ on $\Omega(R), \delta_{m} \in C^{\infty}(\Omega(R))$, supp $\delta_{m} \subset \Omega(R)$, and $\delta_{m} \underset{m \rightarrow \infty}{\longrightarrow} \delta(x-y)$ in the sense of distributions over $C^{\infty}(\Omega(R))$. Then in the same sense $h_{m}(x, y) \rightarrow$ $\Gamma_{R}(x, y)$, where $\Gamma_{R}(x, y)$ is the Green function of the Dirichlet problem on $\Omega(R)$. Let $g_{m}=h_{m} \eta_{\varepsilon \tau}(t)$, where $\varepsilon \in(0, T), \tau \in(0, T-\varepsilon)$. Then from (12), where $g=g_{m}, \theta=0, G(\theta)=0$ we have

$$
\int_{\Omega(R)} \delta_{m} d x \int_{0}^{\tau} G\left(u_{R}\right) d t \leq \int_{\Omega(R)} \rho \varphi h_{m} d x .
$$

Passing to the limit as $m \rightarrow \infty$ we obtain that for all $y \in \Omega$

$$
\int_{0}^{\tau} G\left(u_{R}(y, t)\right) d t \leq c_{n} \int_{\Omega} \frac{\rho(x) \varphi(x)}{|x-y|^{n-2}} d x,
$$


where $c_{n}$ depends on $n$ only. Suppose $r \in\left(R_{0}, R\right)$. Then by (4) and (20) for any $y \in \Omega(r)$ we have

$$
\int_{0}^{T} G\left(u_{R}(y, t)\right) d t \leq c(r) .
$$

Using the comparison principle we obtain that $u_{R}$ (and consequently $G\left(u_{R}\right)$ ) is a nondecreasing function of $R$ for $(x, t) \in Q_{T}$. Now from (21), (7) we can obtain that a.e. on $Q_{T}, u_{R} \rightarrow u \in \widetilde{L}_{\mathrm{loc}}^{1}\left(Q_{T}\right)$ as $R \rightarrow \infty, G(u) \in \widetilde{L}_{\mathrm{loc}}^{1}\left(Q_{T}\right)$. Moreover, $G(u) \in L^{1}(0, T)$ and (13) is valid. It follows from the lemma and (4), (20) that function $u$ satisfies condition (8). Suppose $g \in \tilde{C}_{0}^{\infty}\left(Q_{T}\right)$ and supp $g \subset Q_{T}(R)$. Passing to the limit as $R \rightarrow \infty$ in (12), where $G(\theta)=0$, we obtain (14). It is obvious now that condition $C$ is valid and $u$ is a minimal solution. Q.E.D.

Remark 2. Putting, as above, $g=g_{m}, \theta=0$ into (12) and passing to the limit as $\varepsilon \rightarrow T-\tau, m \rightarrow \infty$, we obtain that $u(\cdot, \tau)$, where $\tau \in(0, T)$, belongs, like $\varphi$, to the class of functions summable over $\Omega$ with the weight $\rho|x|^{2-n}$. Generally speaking there is no uniqueness of solutions in this class (see [4]).

The uniqueness we shall prove in the class of functions satisfying the following condition at infinity

$$
\lim _{R \rightarrow \infty} R^{-n} \int_{\Omega(R, 2 R)} d x \int_{0}^{T} G(u) d t=0,
$$

which is slightly weaker than (8).

Theorem 2. Let the conditions of Theorem 1 be satisfied, with the possible exception of (4). Then the problem (1), (2), (3) has only one solution, satisfying condition (22).

Proof. Suppose $v$ is a solution of our problem satisfying (22). Due to (15), a.e. on $Q_{T}(R)$

$$
u_{R} \leq v .
$$

The function $u_{R}$ is nondecreasing with respect to $R$ and $v \in \widetilde{L}_{\text {loc }}^{1}\left(Q_{T}\right)$. Now we obtain from (23) that $u_{R} \rightarrow u \in \tilde{L}_{\text {loc }}^{1}\left(Q_{T}\right)$ a.e. on $Q_{T}$ as $R \rightarrow \infty$, where $u$ is a minimal solution of the problem (1), (2), (3), satisfying (22). It remains to prove that $v=u$. By Definition 1

$$
\int_{Q_{T}}\left((G(v)-G(u)) \Delta g+(v-u) \rho g_{t}\right) d x d t=0 \quad \forall g \in \tilde{C}_{0}^{\infty}\left(Q_{T}\right),
$$

where $v-u \geq 0, G(v)-G(u) \geq 0$ a.e. on $Q_{T}$. Let us choose some smooth function $f: \Omega \rightarrow \mathbb{R}_{+}, f \not \equiv 0$, supp $f \subset \Omega$. Let $h$ be a solution of the problem

$$
\begin{gathered}
\Delta h=-f, \quad x \in \Omega, \\
\left.h\right|_{\partial \Omega}=\left.h\right|_{\infty}=0 .
\end{gathered}
$$

Then $h \geq 0$ on $\Omega$ and for $s=0,1$

$$
\left|\frac{\partial^{s} h}{\partial x_{j}^{s}}\right| \leq C(1+|x|)^{2-n-s}, \quad j=1, \ldots, n,
$$


where $x=\left(x_{1}, \ldots, x_{n}\right) \in \Omega$. Suppose for $R>R_{0}, \varepsilon \in(0, T)$, and $\tau \in$ $(0, T-\varepsilon)$,

$$
g_{R}(x, t)=h(x) V_{R}(r) \eta_{\varepsilon \tau}(t) .
$$

Then $g_{R} \in \widetilde{C}_{0}^{\infty}\left(Q_{T}\right)$. Putting $g=g_{R}$ in (24) we have

$$
\begin{aligned}
\int_{\Omega(2 R)} & f V_{R} d x \int_{0}^{T-\varepsilon}(G(v)-G(u)) \eta_{\varepsilon \tau}(t) d t \\
= & \int_{Q_{T}}(v-u) \rho h V_{R} \eta_{\varepsilon \tau}^{\prime} d x d t \\
& +\int_{\Omega(R, 2 R)}\left(2 \nabla h \nabla V_{R}+h \Delta V_{R}\right) d x \int_{0}^{T-\varepsilon}(G(v)-G(u)) \eta_{\varepsilon \tau}(t) d t
\end{aligned}
$$

The integral over $\Omega(R, 2 R)$ tends to zero as $R \rightarrow \infty$ by (22) and (27). Since $\eta_{\varepsilon \tau}^{\prime} \leq 0$ we get from $(28)$ that $G(v)=G(u)$ a.e. on the set $\operatorname{supp} f \times(0, T-\varepsilon)$. Thus, $v=u$ a.e. on $Q_{T}$. Q.E.D.

From the previous considerations we immediately obtain the following.

Theorem 3. Suppose $\varphi_{j}: \Omega \rightarrow \mathbb{R}_{+}$, all the conditions of Theorem 1 are satisfied for the system $\left(G, \rho, \varphi_{j}\right), j=1,2$, and $\varphi_{1} \leq \varphi_{2}$ a.e. on $\Omega$. Then $u_{1} \leq u_{2}$ a.e. on $Q_{T}$, where $u_{j}$ is a solution of the problem (1), (2), (3) with $\varphi=\varphi_{j}$.

\section{REFERENCES}

1. P. H. Benilan, M. G. Grandall, and M. Pierre, Solutions of the porous medium equation in $\mathbb{R}^{n}$ under optimal conditions on initial values, Indiana Univ. Math. J. 33 (1984), 51-87.

2. H. Brezis and S. Kamin, Sublinear elliptic equations in $\mathbb{R}^{n}$, Manuscripta Math. 74 (1992), 87-106.

3. E. Di Benedetto, Continuity of weak solutions to a general porous medium equation, Indiana Univ. Math. J. 32 (1983), 83-118.

4. D. Eidus, The Cauchy problem for the non-linear filtration equation, J. Differential Equations 84 (1990), 309-318.

5. A. S. Kalashnikov, The Cauchy problem in a class of growing functions, Vestnik Moskov. Univ. Ser. VI, Mat. Mekh. 6 (1963), 17. (Russian)

6. E. S. Sabinina, On the Cauchy problem for the equation of the non-stationary gas filtration, Dokl. Akad. Nauk SSSR 136 (1961), 1034-1037. (Russian)

School of Mathematical Sciences, The Raymond and Beverly Sackler faculty of Exact Sciences, Tel Aviv University, Tel Aviv, IsRael 